\title{
Multidisciplinary Treatment for Headache in the Canadian Healthcare Setting
}

\author{
Khara M. Sauro, Werner J. Becker
}

\begin{abstract}
Background/Objectives: Multidisciplinary treatment programs are seen as an effective way to treat patients with chronic illness. The purpose of this study was to describe a multidisciplinary headache program which was developed in the Canadian public healthcare setting, and to report on patient perceptions of the program and patient treatment outcomes. Methods: The Calgary Headache Assessment and Management Program (CHAMP) was developed with initial funding from Alberta Health, and continued with function from the Calgary Health Region. Patient perceptions of the program were obtained with questionnaires. Outcome measures for a cohort of patients who completed the Self-Management Workshop were obtained using standard headache related disability measures. Results: Patient perceptions of the education session, the Lifestyle Assessment, and the Self-Management Workshop were very positive. Headache Disability Inventory scores fell from 56.2 to 46.3 from baseline to three months post Self-Management Workshop (p<.001). Corresponding scores for the HIT-6 were 63.6 and 58.2 ( $\mathrm{p}<.001)$. Conclusions: Multidisciplinary headache treatment programs can be developed in the Canadian public healthcare system. The program described here was well accepted by many patients and perceived to be useful by them. Headache related disability as measured by standard measures was significantly reduced after participation in the program.
\end{abstract}

RÉSUMÉ: Traitement multidisciplinaire de la céphalée dans le contexte canadien de soins de santé. Contexte/Objectifs : On considère que les programmes multidisciplinaires de traitement sont une façon efficace de traiter les patients atteints de maladies chroniques. Le but de cette étude était de décrire un programme multidisciplinaire de traitement de la céphalée qui a été développé dans le cadre d'un système publique de soins de santé au Canada et de rapporter la perception qu'en ont les patients ainsi que les résultats du traitement. Méthodes : Le Calgary Headache Assessment and Management Program, qui a été développé initialement avec le soutien financier de l'Alberta Health, a continué à fonctionner grâce au soutien de la Calgary Health Region. Des questionnaires ont été utilisés pour connaître l'opinion des patients sur le programme. La mesure des résultats chez une cohorte de patients qui avaient complété l'atelier d'autogesion a été obtenue au moyen de mesures standardisées de l'invalidité reliée à la céphalée. Résultats : L'opinion des patients concernant les sessions d'information, l'évaluation du mode de vie et l'atelier d'autogestion était très positive. Les scores du Headache Disability Inventory ont baissé de 56,2 initialement à 46,3 trois mois après l'atelier d'autogestion (p > 0,001). Les scores correspondants pour le HIT-6 étaient de 63,6 et de 58,2 respectivement (p > 0,001). Conclusions : Il est possible de développer des programmes multidisciplinaires de traitement de la céphalée dans le cadre du système publique de santé au Canada. Le programme que nous décrivons ici a été bien accepté par plusieurs patients et ils sont d'avis qu'il leur a été utile. L'invalidité reliée à la céphalée, telle que mesurée par des outils standards, a été significativement diminuée suite à la participation au programme.

Can. J. Neurol. Sci. 2008; 35: 46-56

Multidisciplinary team-based care is increasingly seen as the best option for treating patients with chronic illnesses. ${ }^{1}$ There is also increasing evidence for the effectiveness of multidisciplinary treatment programs in the chronic pain population. Buchner et al (2006) showed considerable improvement in functionality in patients with chronic low back pain and neck pain after treatment in a multidisciplinary program. Moreover, meta-analyses and systematic reviews have shown the superiority of multidisciplinary pain treatment programs for back pain as compared to wait-list controls, notreatment controls and single-modality treatments., ${ }^{3,4}$ Multidisciplinary treatment has also been shown to be beneficial in the treatment of headache..$^{5,6}$
The success of multidisciplinary treatment may be due in part to the wide range of skills which the team can bring to patient care as compared to those brought by a single healthcare provider. Teams can solve problems that no one individual can

\footnotetext{
From the Department of Clinical Neurosciences, University of Calgary \& Calgary Health Region, Calgary, Alberta, Canada.

Received June 28, 2007. Final Revisions Submitted October 31, 2007. Reprint requests to: W.J. Becker, Division of Neurology, Foothills Medical Centre, 1403 29th St. NW, Calgary, AB T2N 2T9, Canada.
} 
solve. ${ }^{1}$ For headache, there is strong evidence that a number of behavioral and non-pharmacological therapies are effective., Teams are able to bring these therapies to the patient and integrate them with pharmacologic care. Through the extensive use of non-physician health professionals (NPHP) to deliver patient care, multidisciplinary teams have the potential to reduce the physician resources required and create more cost-effective treatment. The patient is an important member of the team, and patient education is an important part of any multidisciplinary treatment program. This education needs to go beyond advice and knowledge, and include mastery of a number of skills which have the potential to assist the patient in the management of a chronic headache disorder.

This article has two objectives. Firstly, we will outline in some detail the structure of our team based multidisciplinary headache program, the Calgary Headache Assessment and Management Program (CHAMP), to demonstrate the type of program that can be developed in the Canadian Healthcare setting. Secondly, we will present data that we have collected on the perceptions that patients have of the CHAMP program, and data on patient outcomes. In this report, we will focus on two aspects of our program evaluation: treatment outcomes for patients completing the Self-Management workshop; and patient perceptions of several of our program components including the Self-Management Workshop, the Education Session and the Lifestyle Assessment. We hope that our experience will promote interest in others to consider the development of multidisciplinary headache programs, and that this account of our experience will facilitate the development of such programs.

\section{MethodS}

\subsection{The Program}

\subsubsection{Program Funding and Staff}

Initial funding for CHAMP was obtained through a competitive process from the Alberta Medical Services Delivery Innovation Fund (MSDIF), which invited proposals for innovative programs designed to deliver health care differently from the conventional patient-physician office consultation. Ultimately, funding was obtained from the MSDIF for salaries for a nurse, an occupational therapist, and a half-time psychologist for three years. Importantly, the program also received a physician alternative payment plan in which physicians were paid a sessional fee (paid by the hour) not only for their direct patient care work, but also for time spent working with the other health professionals in the program, for case discussions and for patient group-education. The program began in September 2003. With time, a part time kinesiologist was added to program staff, and a second nurse was funded by the Calgary Health Region (CHR).

After three years, CHAMP was evaluated by the CHR, and long term funding for salaries was provided to the program by the CHR Regional Pain Program. The physician sessional alternative payment plan was continued by Alberta Health. At three years, five neurologists provide services to the program on a part time basis.

From the start of the program, the CHR provided the necessary clerk resources to book physician appointments, etc, and also provided clinic space.

\subsubsection{Program Design}

The core of the program consists of five pillars (Table). All patients must be referred by a physician, and are triaged, based upon the referral letter. Where significant diagnostic concerns or urgent considerations exist, patients are expedited directly to the physician consultation. Patients with cluster headache and patients from out of town, where distance makes attendance at the various program components difficult, are booked to see the physician directly. For the majority of patients, however, entry to the program occurs through the patient Education Session. Attendance at the Education Session is mandatory before the physician appointment is booked, with the rational that if patients are unwilling to invest some of their own time and energy in their headache management, they are unlikely to make use of the CHAMP interdisciplinary team. The referral letters of patients who do not attend the Educations Session (with the exceptions listed above) are returned to the family physician with the understanding that if necessary they can be referred to a general neurologist's office. Once the patients have attended the Education Session they are registered in the program, and are able to sign-up for workshops, lectures, and a Lifestyle Assessment. They are also put on the waiting list to see a physician. Patient flow through the program is summarized in Figure 1.

The various components of CHAMP are described in more detail below:

The Education Session: To make attendance at the Education Session convenient, three sessions are scheduled per month on different days of the week, with sessions in the morning, afternoon, and evening. These two hour sessions are taught by a physician and either an occupational therapist or a psychologist. The first hour focuses on headache diagnosis, pathophysiology, and management. Significant time is spent on medication overuse headache, and much of the presentation focuses on migraine as this is the most common headache type referred to the program. The second hour focuses on behavioral aspects of headache management. These include headache trigger identification and management, lifestyle adjustments to promote

Table: The 5 pillars of CHAMP

\section{The Five Pillars of the CHAMP Program}

1. Education Session

2. Lifestyle Assessment

3. Self-Management Workshop

4. Nursing Contact and Advice

5. Physician Visit

Each pillar represents a different aspect of the multidisciplinary program 


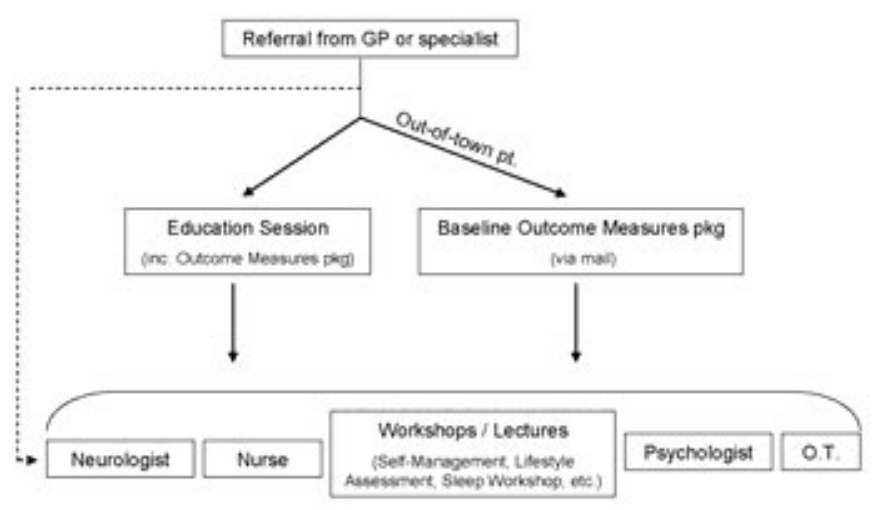

Figure 1: Patients are referred to CHAMP by a general practitioner or a specialist (i.e. Neurologist). They are then registered in the mandatory Education Session. There are two exceptions to this process. If patients are from outside the Calgary area they are given the option to not attend the Education Session, are mailed an outcomes measure package and are booked to see a CHAMP Neurologist. These patients do have access to all of the program components. Also, if patients are deemed, via a triage procedure, to need an immediate visit with the Neurologist they are 'fasttracked'. After the Education Session patients are encouraged to participate in the NPHP-run workshops and lectures while they are on a waiting list for a Neurologist visit.

better headache control, relaxation techniques, and cognitive behavioral therapy with a major emphasis on the importance of stress management. The Education Session also explains the various components of CHAMP, and patients are encouraged to sign up immediately for other program components including the Lifestyle Assessment and the group workshops. Patients are able to make use of all the non-physician CHAMP resources before they see the physician. While waiting to see the physician patients can utilize this time to become more knowledgeable about headache, and to work on the non-medical aspects of headache management. This approach was felt to be safe, as all patients are referred to the program by a physician and therefore have already received some medical attention. Calgary Headache Assessment and Management Program staff will expedite the physician assessment at any time if this seems appropriate.

The Lifestyle Assessment: Although the Education Session briefly explores how attention to life-style issues such as regular sleep and meals can influence headache patterns, and the importance of avoiding specific migraine triggers, the Lifestyle Assessment provides a more in depth exploration of these issues in an individual session with a NPHP. The Self-Management group workshops also explore some of these issues, but some patients do not attend these workshops for a variety of reasons, including time constraints, and a reluctance to become involved in group sessions with other patients. The Lifestyle Assessment explores ways for the patient to reduce headache frequency through lifestyle modification. Specific headache triggers are discussed, as well as dietary habits, sleep quality, exercise, posture, psychosocial factors, and stress. Areas of concern are identified and recommendations made.
The Self Management Workshop: This workshop is in many ways the core of the NPHP part of CHAMP. Its goal is to teach behavioral skills that can facilitate headache self management. It consists of five, two hour sessions which take place once a week for five consecutive weeks. Patients are encouraged to be active participants in the workshop. They are given hand-outs and homework to encourage incorporation of the techniques taught in the workshop into their daily lives. The goal of SelfManagement is to help patients develop the skills and knowledge required to take ownership of their headache pain; in other words, to take control of their headache pain rather than have it take control of them. This workshop emphasizes a stress-pain connection and stress management is a focus throughout. The multidimensional aspect of pain is emphasized and strategies addressing the cognitive, emotional and physical aspects of headache pain management are introduced. The first session is intended to establish a sense of comfort within the group, and focus on reinforcing that self-management of headache pain is attainable and that the patient has the ability to alter the headache experience. Learning self-monitoring of the stress response helps patients to understand that thoughts and emotions can trigger or worsen a headache. The basics of relaxation and breathing are also introduced. The homework assigned involves keeping a stress $\log$ and practicing diaphragmatic breathing. Different relaxation techniques are introduced in each of the sessions, including visualization, progressive muscle relaxation, autogenics, and biofeedback. The second session focuses on the concept of activity pacing and lifestyle balance. Seven steps to effective pacing are outlined: identifying responsibility, prioritizing responsibilities/tasks, setting average tolerance for each responsibility, creating schedules, adhering to set schedules, slowly increasing tolerance for activities, and defining rest periods. The balance between productive activity, leisure activity, and self-care activity is emphasized. To reinforce these concepts, patients are asked to record their daily activities over the next week and to reflect on how their current approach to activity may trigger headaches. In the third session patients are given an overview of the Gate Control Theory of pain ${ }^{8,9}$ as in introduction to the cognitive modulation of pain and the individuality of the pain experience. Patients are taught cognitive restructuring through identifying negative thoughts, evaluating the usefulness of the thoughts, and examining ways to challenge and change negative thoughts. The homework for this session is to record a minimum of three automatic thoughts and to consider how to change those thoughts. The fourth session focuses on communication style. The impact that negative communication styles can have on relationships and how this can consequently increase stress and have a detrimental effect on headache pain, is reinforced. Patients are taught key aspects of effective communication, such as expressing intentions clearly; expressing needs, wants and feelings clearly; and actively listening to others. Patients are asked to use communication techniques taught in the workshop to re-work an interaction they were not content with. The final session, session five, involves the creation of coping plans. Key questions considered in the development of a coping plan include: What is the difference between a 2/10 headache vs $8 / 10$ headache? What are my cues that a headache is beginning? What triggers/situations often lead to a headache for me? What coping strategies work for me? What 
external supports can help me deal with my headaches and how? Patients are encouraged to include many different strategies and treatments (pharmacological and non-pharmacological) in their coping plans.

Nursing: Aside from general clinic coordination which the nurse does with the help of clerks who book the appointments and laboratory tests, the nurse performs two other important functions. Firstly, the nurse is the telephone contact point for patients in the program if they encounter significant medication side effects or require medical advice. Secondly, the nurse plays an active role during the physician visits; providing medication and general headache teaching, and helping to obtain the patient history. The nurse is assisted in this work by standardized medication sheets which outline the usual doses for medications, and potential side effects.

Physician Visits: The medical assessments in CHAMP are similar to traditional medical assessment of headache patients. Care is focused on formulation of a specific headache diagnosis or diagnoses. In CHAMP, patients are followed up more than in a typical neurology consultation practice; most patients have several follow-up visits over the course of the first year and are followed longer if significant disability persists.

Other Program Components: The additional components include the Body Works workshop which consists of three sessions led by a Kinesiologist. Its goal is to assist patients in maintaining good posture, and to learn neck strengthening and stretching exercises in order to minimize any headache triggers related to poor posture and neck pain. The Sleep Workshop's goal is the reduction of headache through promotion of better sleep. It focuses on patient education about sleep hygiene. Other resources include a Relaxation Workshop, and lectures on topics related to headache management. Some one-on-one psychological assessment and counseling is also available to patients in CHAMP, as is counseling from an occupational therapist with regard to ergonomic and other issues.

\subsection{Outcome Measurement}

Since its inception, the CHAMP program has collected a number of outcome measurements in order to facilitate program development. The program has been reviewed by the Conjoint Health Research Ethics Board of the University of Calgary. At the Educations Session, patients complete a number of measures which serve as a baseline for future comparison. For out of town patients, the baseline outcome measures are mailed in advance of their physician appointment, and completed at home. These measures include the HIT-6, HDI, CES-D, and VAS based measures of pain and suffering due to pain. These measures are also routinely administered to patients during their time in the program, particularly in relation to their participation in the SelfManagement workshop. In addition, program specific questionnaires are completed by patients to assess how well various components of the program met their needs.

\section{Headache Disability Inventory (HDI)}

The $\mathrm{HDI}^{10}$ is a self-administered, 40 item inventory designed to assess the perceived amount of disability attributed to headache. The HDI has two subscales; the functional subscale and the emotional subscale. Patients are asked to respond to statements regarding their headaches (i.e. I do not enjoy social gatherings because of my headaches) using a 3 point Likert type scale: 'Yes', 'Sometimes', and 'No'.

\section{Headache Impact Test (HIT-6)}

The HIT- $6^{11}$ is a six item self-administered questionnaire which measures the impact of headache on the patient's ability to function at work/school, at home, and in social settings over the past four weeks. Patients are asked to respond 'Never', 'Rarely', 'Sometimes', 'Very Often', or 'Always' to statements regarding how their headaches affect their life (i.e. In the past four weeks, how often have you felt fed up or irritated because of your headaches?).

\section{Center for Epidemiological Studies - Depression Subscale Revised (CES-DR)}

An abbreviated form (11-item self-report questionnaire) of the CES-D was used to assess symptoms of depression within the past week. ${ }^{12}$ Patients are asked to indicate how often they experienced certain symptoms identified by statements such as 'I felt that everything I did was an effort' using the options provided.

\section{Headache Frequency \& Intensity}

Patients were asked to record the number of days, in the past month, that they had a headache. They were also asked to rate, on a VAS, the average intensity of their headaches. The Intensity VAS was anchored on the left with 0 (no pain) and on the right with 10 (the most intense pain imaginable).

\section{Suffering Due to Headache}

A similar VAS was used by the patients to rate the amount of suffering they experienced, in the past month, due to their headaches. The patients rated their suffering on an 11 point VAS anchored on the left with 0 (no suffering) and on the right with 10 (extreme suffering).

\subsubsection{Evaluation of the Self-Management Workshop}

Patients were asked to complete two brief 10 item questionnaires related to their experience in the SelfManagement workshop. Questionnaire items were either an 11 point, Likert-type Visual Analogue Scales (VAS) or forced response questions.

The first evaluation was completed at the end of the last session of the Self-Management workshop. Patients completed the evaluations and placed them in an envelope to assure confidentiality. This evaluation was primarily an evaluation of the Self-Management workshop materials and facilitators. The questions asked patients to rate the amount of benefit they gained from participation in the workshops, and for their assessments of the relevance, and usefulness of its various components. They were also asked to rate the overall effectiveness of the workshops, and how likely they thought it was that they would use the various skills and techniques taught in the workshop.

The second evaluation was mailed to participants three months following completion of the workshop. Participants were asked to complete the evaluation and return it, anonymously. This evaluation addressed whether the patients continued to use the skills taught in Self-Management. Patients answered each 
question using an 11-point Likert Scale anchored on the left with zero and on the right with ten. The specific questions addressed were: 1$)$ the degree of improvement in their ability to cope with headaches $(0=$ no improvement and $10=$ substantial improvement), 2) the degree to which the self-management techniques remained relevant to them, 3) degree of change in awareness of how thoughts and feelings affect headache, 4) their ability to identify stressors, 5) the frequency of use of pacing techniques, 6) change in awareness of communication style, and 7) frequency of use of relaxation techniques. The questionnaire also asked participants to indicate which of the techniques taught in Self-Management were the most useful in managing their headaches.

In addition, patients attending the Self-Management workshops were asked to complete the outcome measures (HIT-6, HDI, CES-D, and VAS based measures of pain and suffering) directly after completion of their last self management workshop, and three months later.

\subsubsection{Evaluation of the Education Session}

At the end of the two hour education session participants were asked to fill out a brief questionnaire to determine if the objectives of the education session were met. A forced-choice, yes or no, question asked if they would recommend the education session to other headache suffers.

\subsubsection{Evaluation of the Lifestyle Assessment}

Patients completed a simple questionnaire after attending the Lifestyle Assessment. One of the questions asked them to indicate from a list of the topics covered which topics were most relevant to them. Another asked if they would recommend participation in the Lifestyle Assessment to others with difficult headache problems.

\subsection{Statistical analysis}

Statistical analysis was done using SPSS 11.5 (SPSS Inc., Chicago, IL, USA). Descriptive statistics including mean, standard deviation, and frequencies were collected to aid in identifying which aspects of the Self-Management workshop and Lifestyle Assessment were most useful in managing headaches. Frequencies were tabulated to examine the number of patients that would recommend the Education Session, the Lifestyle Assessment and the Self-Management workshop to others suffering for headaches.

Paired t-tests were used to examine differences in the means of pain and disability measures between baseline and following Self-Management. For all comparisons, differences were considered significant if the probability value obtained (p) was .05 or less.

\section{RESUlts}

\subsection{Evaluation for Patients Completing the Self-} Management Group Workshop

\subsubsection{Subjects}

Data from 139 patients, who participated in the SelfManagement workshop during 2004 and 2005, were analyzed. Seven patients $(5 \%)$ were excluded from the analysis because of incomplete attendance. The remaining patients $(\mathrm{N}=132)$ attended at least half of the five Self-Management classes (3 out of 5 or greater); the mean number of classes attended was $4.2( \pm$ $\mathrm{SD}=0.92)$. Eighty seven per cent $(\mathrm{N}=115)$ were female. The mean age of the participants was 44.5 years of age $( \pm$ SEM $=.90)$.

The most common diagnosis among patients, according to the International Headache Society (HIS) criteria, was migraine without aura $(37 \% ; \mathrm{N}=51)$; followed by medication overuse headache $(28.3 \% ; \mathrm{N}=39) .{ }^{13}$ Other diagnoses included chronic migraine $(18.1 \%, \mathrm{~N}=25)$, migraine with aura $(7.2 \%, \mathrm{~N}=10)$, tension type headache $(4.3 \%, \mathrm{~N}=6)$, post-traumatic headache $(4.3 \%, \mathrm{~N}=6)$, and new daily persistent headache $(0.7 \%, \mathrm{~N}=1)$. The most common secondary diagnosis was migraine without aura $(43.5 \%, \mathrm{~N}=60)$. Approximately $47 \%$ of our patients would have met diagnostic criteria for transformed migraine, an older diagnostic category not included in the IHS classification. ${ }^{14}$ There was no correlation between diagnosis and percent decrease in headache days per month $(\mathrm{r}=.151)$.

The mean number of headache days in the month preceding participation in the Self-Management workshop as estimated by the patients was 18.1 days $( \pm$ SEM $=.90)$. The mean pain intensity of these headaches was $5.7( \pm$ SEM $=.17)$. This suggests that patients were experiencing headaches of at least moderate intensity most days of the month.

\subsubsection{Workshop Evaluations}

The evaluation questionnaire administered immediately following the Self-Management workshop was returned by 115 of the 132 participants in the workshop (86.5\%). When asked how beneficial they felt the workshop was, the mean VAS rating was $8.29( \pm \mathrm{SEM}=.15)$ out of a possible 10 . Seventy-four $(64 \%)$ of the participants rated the workshop as being of 'maximum benefit' (a VAS of 8 -10). When the participants were asked if they believed they would continue to use the skills they were taught in the workshop, $79.5 \%$ of patients said they were 'very likely' to continue to use the self-management techniques and nearly all of the remaining patients $(17.82 \%)$ reported being 'somewhat likely' to do so.

The three month post Self-Management workshop questionnaires were returned by $53.57 \%(\mathrm{~N}=61)$ of the patients. The majority (95\%) reported using the self-management techniques either 'Occasionally' or 'Routinely'. The mean VAS rating for this item was $6.87( \pm \mathrm{SD}=1.71)$ suggesting that patients were using the self-management techniques frequently (Figure 2).

Using the Likert Scales, on average patients reported that the Self-Management workshop improved their ability to cope with their headaches $($ mean $=6.48 \pm \mathrm{SD} 2.07)$; that the topics covered in the Self-Management workshop continued to be relevant (mean $=7.47 \pm \mathrm{SD} 2.22)$; that after the Self-Management workshop their awareness of how their thoughts/feelings impact their headaches was significantly increased (mean $=7.91 \pm \mathrm{SD}$ 1.73 ); that their ability to identify stressors in their life since the workshop increased $($ mean $=7.71 \pm$ SD 1.77$)$; that they frequently used the activity pacing techniques they were taught in the workshop (mean $=6.92 \pm$ SD 2.14); that their awareness of their communication style and that of those around them had increased $($ mean $=5.76 \pm$ SD 2.61); and that they frequently used relaxation techniques taught in the Self-Management workshop $($ mean $=5.44 \pm \mathrm{SD} 2.8)($ Figure 3$)$. 


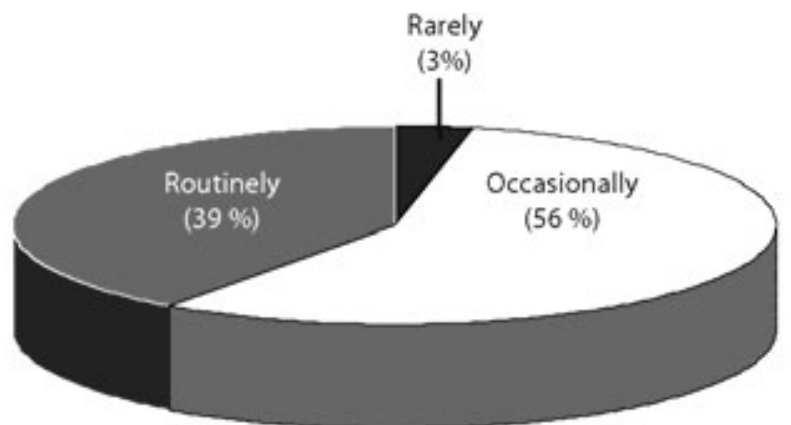

Figure 2: Most patients reported continuing to use the self-management techniques occasionally or routinely three months following the workshop.

The three month post-workshop evaluations also explored the relative usefulness of the various self management techniques as assessed by the patients. Of the seven self management modalities assessed, relaxation techniques, pacing and stress management were felt by the patients to be the most useful (Figure 4).

\subsubsection{Outcome Measures}

Our primary analysis was a comparison between our baseline outcome measures collected at the Education Session prior to the

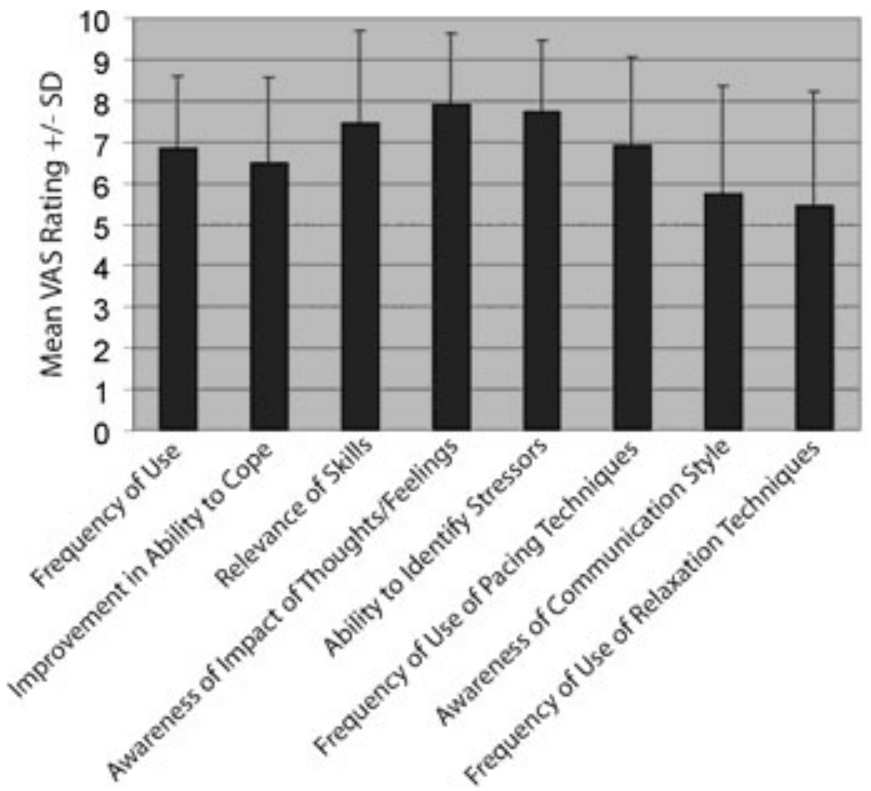

Figure 3: Using an eleven point, Likert-type scale anchored with 0 and 10, patients rated the usefulness of the Self-Management tools. Three months following the Self-Management workshop patients reported that they were still utilizing the tools which they were taught in the SelfManagement workshop. Furthermore, they reported that the skills they were taught were still very relevant three months after the workshop and the techniques taught in the workshop continued to be of benefit in managing their headaches. patients starting the Self-Management workshop, and the values for the same outcome measures at three months post SelfManagement workshop completion.

Not all patients returned the three month post- SelfManagement workshop questionnaire. For these patients $(\mathrm{N}=$ 27) data from the post- Self-Management time-point was brought forward and used for the three month post- SelfManagement outcomes allowing data analysis for 112 patients. To ensure that this method of analysis did not bias our results we conducted a sub-analysis on 31 patients with data available at both immediately post- Self-Management and three month postSelf-Management. This analysis on average showed greater improvement three months following Self-Management than immediately following completion of the Self-Management workshops. This suggests that using the data available immediately after Self-Management for those patients where data was not available at three months after Self-Management biased our results in a negative fashion. Our method of analysis would therefore tend to minimize the amount of improvement found rather than to exaggerate it.

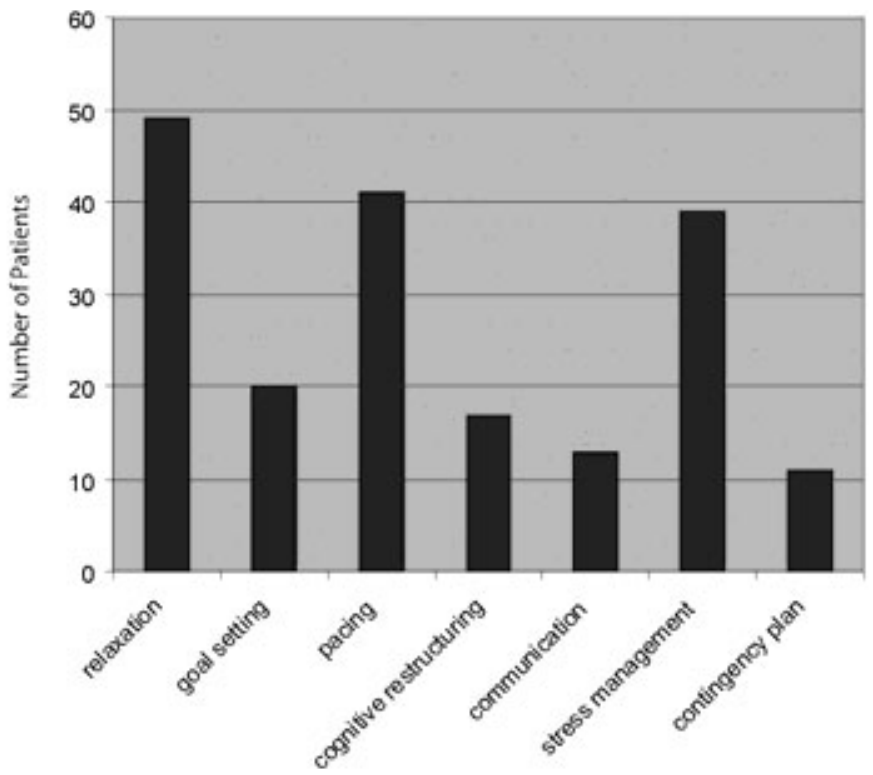

Figure 4: When asked which Self-Management techniques were most useful, patients found techniques associated with stress reduction (relaxation, activity pacing, and stress management) to be the most helpful.

\section{Headache Disability Inventory (HDI)}

The HDI scores showed an improvement in headache disability following Self-Management $(\mathrm{N}=102)$. The amount of disability due to headaches, as measured by the HDI was found to be significantly less post- Self-Management $($ Mean $=46.3 \pm$ 
a)

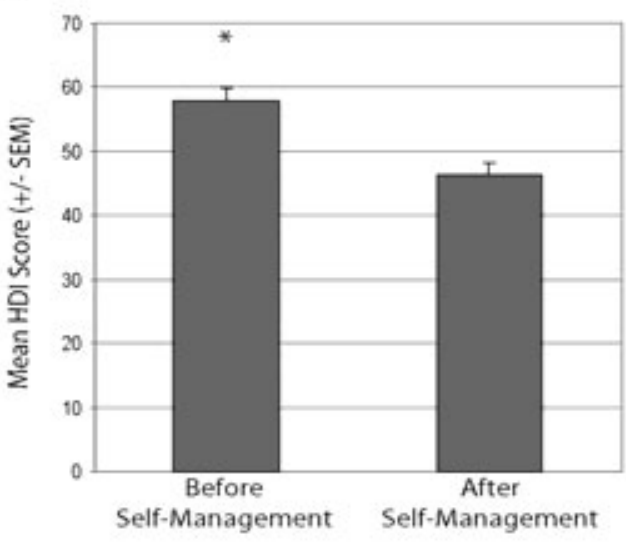

D]

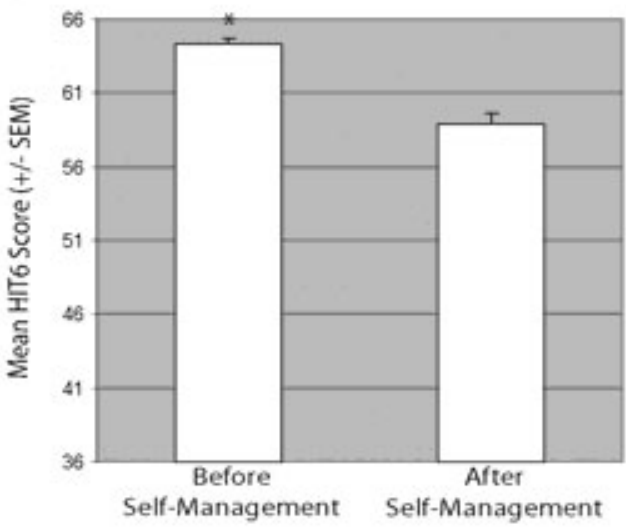

Figure 5: a) Following Self-Management workshop, patients $(N=102)$ reported significantly less disability associated with their headaches than before Self-Management, as measured by the Headache Disability Index $(t(1,92)=4.749 ; p<.001)$. b) Following Self-Management the impact reported by patients $(N=105)$ that headache had on their life, as measured by the Headache Impact Test, decreased significantly (t $(1$, 98) $=7.549 ; p<.001)$.

$\mathrm{SEM}=2.2)$ as compared to baseline $($ Mean $=56.2 \pm \mathrm{SEM}=2.1)$ $(t(1,92)=4.749 ; \mathrm{p}<.001)$ (Figure 5a). Furthermore, scores for the two subscales; the functional and emotional subscales of the HDI, were both found to be significantly lower post- SelfManagement as compared to baseline $(t(1,92)=3.925 ; \mathrm{p}<.001$ and $t(1,92)=4.422 ; \mathrm{p}<.001$, respectively $)$.

\section{Headache Impact Test (HIT-6)}

The analysis of the HIT-6 scores demonstrated that following Self-Management, headaches had less impact on daily functioning $(\mathrm{N}=105)$, with HIT-6 scores decreased postworkshop as compared to baseline. Mean HIT-6 scores at baseline were $63.6( \pm \mathrm{SEM}=.5)$, as compared to $58.2( \pm \mathrm{SEM}=$ .9) post self management completion $(t(1,98)=7.549 ; \mathrm{p}<.001)$ (Figure 5b).

\section{Headache Frequency \& Intensity}

Both the number of headache days and the intensity of headaches decreased following Self-Management $(\mathrm{N}=99)$. The mean number of days with headache prior to participation in the Self-Management workshop was 18 days as compared to 12 days following Self-Management $(t(1,84)=6.141 ; \mathrm{p}<.001)$ (Figure $6 a)$. The average intensity of the headaches decreased from 5.5 $( \pm \mathrm{SEM}=.2)$ at baseline to $5.1( \pm \mathrm{SEM}=.2)$ post-workshop $(t(1$, $89)=2.202 ; \mathrm{p}<.001)($ Figure 6b)

\section{Center for Epidemiological Studies and Suffering Due to Headache}

Depressive symptoms were also improved following SelfManagement $(\mathrm{N}=100)$. Symptoms of depression as measured by the CES-D scores decreased significantly following the SelfManagement workshop (Mean $=7.5 \pm \mathrm{SEM}=.7$ ) as compared to baseline $($ Mean $=9.5 \pm \mathrm{SEM}=.7)(t(1,87)=3.703 ; \mathrm{p}<.001)$. This can be seen in Figure 7a. Similarly, the amount of suffering

a)

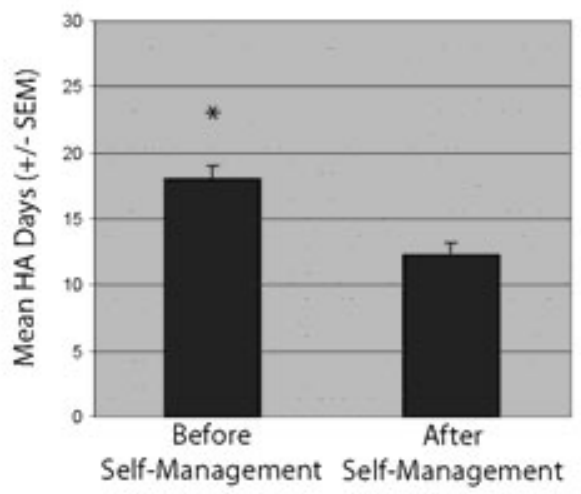

b)

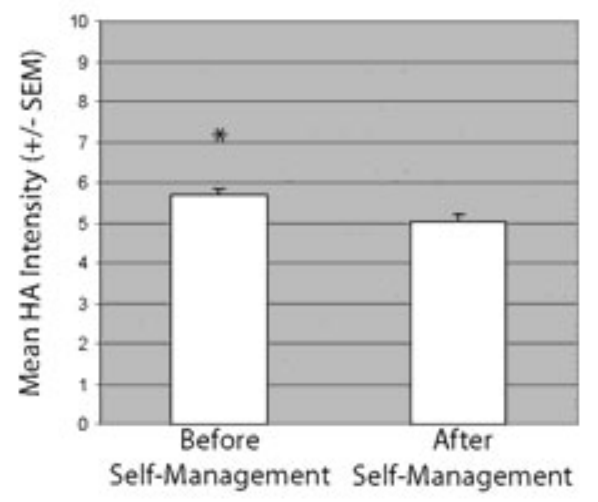

Figure 6: a) Patients $(N=99)$ suffered from headaches fewer days per month following the Self-Management workshop compared to before $(t(1,84)=6.141 ; p<.001)$. b) The remaining headaches that the patients $(N=99)$ experienced following Self-Management workshop were less severe, as measured by a eleven point VAS, than before the workshop $(t(1,89)=2.202 ; p<.001)$ (Figure 6b). 
a)

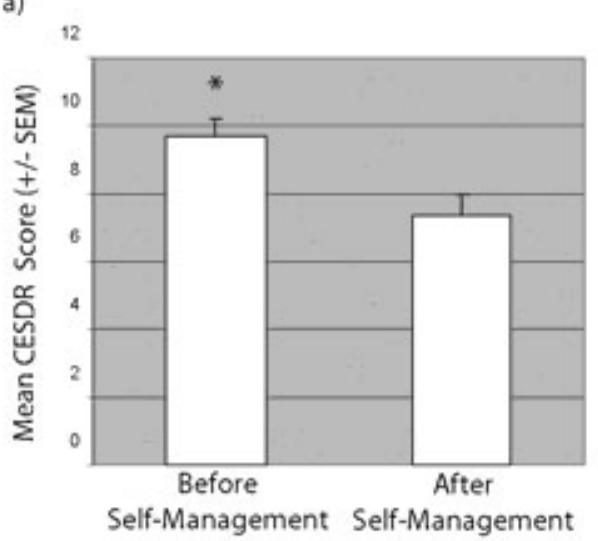

b)

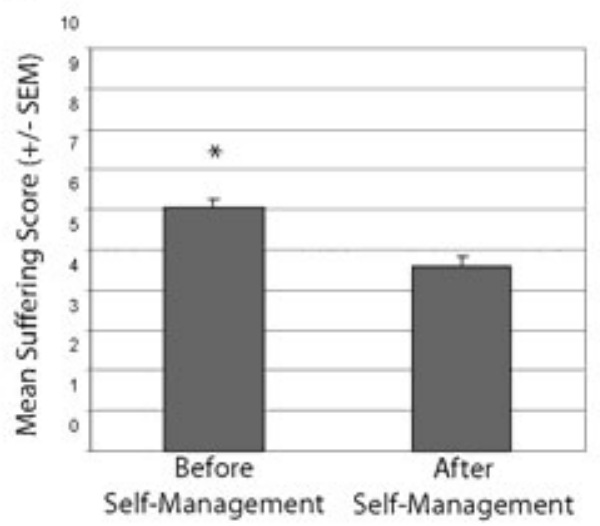

Figure 7: a) Following the Self-Management workshop patients $(N=$ 100) had significantly less depressive symptoms, as measured by CES-D) than before Self-Management $(t(1,87)=3.703 ; p<.001)$. b) Following the Self-Management workshop patients reported significantly less suffering from their headaches, as measured by an eleven-point VAS, than before the Self-Management workshop $(t(1,89)=5.697 ; p<.001)$.

due to headaches decreased post-workshop as compared to baseline values $(t(1,89)=5.697 ; \mathrm{p}<.001)($ Figure $7 \mathrm{~b})$.

\subsection{Evaluation of the Education Session}

Evaluations completed by all patients who participated in the Education Session in 2004 were analyzed $(\mathrm{N}=194)$.

When asked how well the objectives of the Education session were met, all patients that participated in the Education Session found that all the objectives of the session were "very well met". When patients were asked if they would recommend the Education Session to others suffering from headaches, of those that responded $99.4 \%(\mathrm{~N}=171)$ said they would recommend this session. Only one person (.6\%) said they would not recommend it.

\subsection{Evaluation of the Lifestyle Assessment}

Data was collected from 60 consecutive patients who took part in a lifestyle assessment during 2005 and 2006. Of the eight

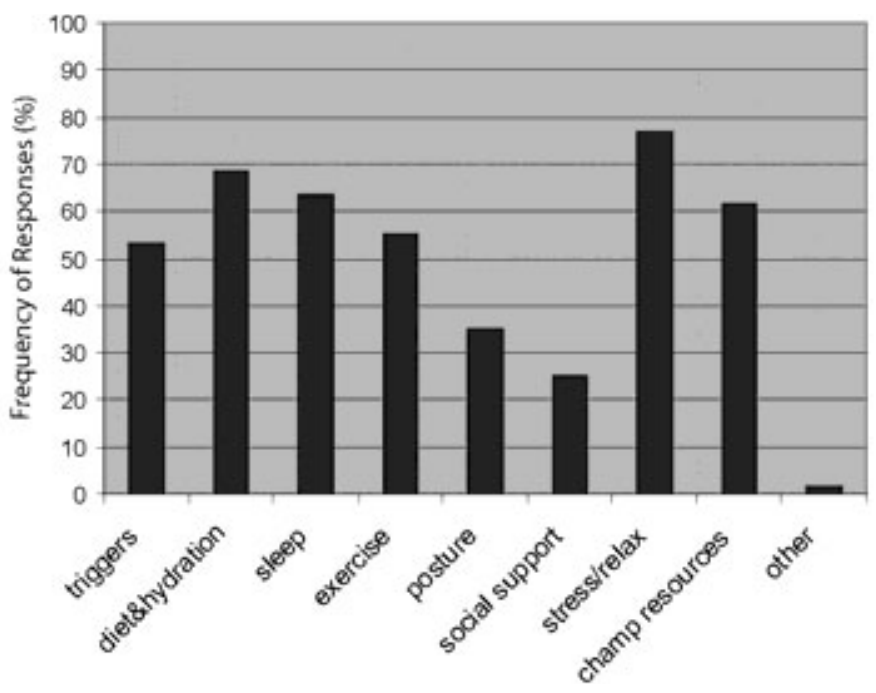

Figure 8: Stress \& Relaxation, Diet \& Dehydration, and Sleep were deemed by the patients to be the most relevant of the lifestyle topics discussed. The explanation of the CHAMP resources was also found to be very useful.

topics discussed during the lifestyle assessment interview, stress management, diet, and sleep were considered by patients to be the ones that were most relevant to their situation (Figure 8). However, other topics were also considered relevant by many patients, depending upon individual circumstances. When asked if they would recommend the lifestyle assessment to other patients suffering from headaches, all patients recommended participation in the lifestyle assessment.

\section{DisCUSSION}

The experience of CHAMP provides evidence that, with appropriate support from funding agencies, a multidisciplinary headache program can be successfully established as part of the Canadian public healthcare system. Our analysis showed that the patients who participated in the multidisciplinary components of our program felt very positive about their experience. Virtually all patients who participated in the Education Session and the Lifestyle Assessment indicated that they would recommend these to others. Those portions of the Lifestyle Assessment which most patients felt were most useful to them dealt with stress management, diet, and sleep hygiene, all topics which appropriately trained non-physician healthcare professionals can deal with; perhaps more efficiently than many physicians. Likewise, the great majority of patients who completed the SelfManagement workshop felt they had benefited from their participation. Furthermore, three months after completion of the Self-Management workshop most were still using the skills they had learned in the workshop, with many using them routinely. The Self-Management skills which patients found most useful included activity pacing, stress-management, and relaxation techniques. This finding is consistent with prior studies suggesting that stress is a major trigger of migraine headaches. ${ }^{15}$ 
Some other skills, such as cognitive restructuring and contingency planning were seen as less useful. This is somewhat surprising, as cognitive behavioural therapy has been shown to be effective in headache management. ${ }^{16-19}$ However, it is possible that the cognitive techniques taught in the selfmanagement workshop were too complex for the amount of time allocated to these techniques. Also, many patients reported that they failed to create contingency (or flare-up) plans, perhaps because of time constraints. Because many of the patients in the present study work full-time and have many responsibilities, the length of the CHAMP Self-Management workshops was limited to two hours a week for five weeks. For some patients, this may have been too short for optimum results.

The outcome measures, which focused on improvement in headache days, intensity, suffering, and disability, confirmed overall improvement in the patient group on all measures examined. The number of headache days per month was reduced from a mean of 18 days at baseline to 12 days after SelfManagement completion. Although it is difficult to translate the mean reduction in headache frequency of six days a month into actual improved functionality and quality of life for our patients, intuitively, six additional headache free days would be expected to make a difference in the functionality of our patients. This is supported by the observed mean reduction in HDI scores, a measure of headache related disability, of ten points following Self-Management. Furthermore, HIT6 scores also showed improvements after Self-Management from baseline. Although the clinical significance of the reduction in HIT -6 scores and HDI scores experienced by our patients during their time in the program is not clear, the simultaneous reductions that occurred in our clinical outcome measures (headache frequency, headache intensity, suffering experienced as a result of headache, etc) would suggest that our patients did indeed experience a clinically significant improvement. The majority of patients also indicated on their Self-Management evaluations that they were better able to cope with their headaches after completing the SelfManagement workshop. These results are consistent with the few studies which have examined the effectiveness of multidisciplinary treatment programs for headache. ${ }^{5,6}$ For example, a randomized study comparing a multidisciplinary program, focused on supervised group exercise therapy sessions, to standard care with the patient's family physician found significantly better outcomes in the multidisciplinary group on a number of outcome measures including headache frequency, and pain related disability. ${ }^{6}$ Similarly, Scharff \& Marcus (1994) compared a patient group who completed a multidisciplinary program; five three-hour weekly sessions with a neurologist, a physical therapist, an occupational therapist, and a psychologist, to a control group of patients who for a variety of reasons were unable to attend the multidisciplinary program. Significant improvements were reported in the treatment group as compared to the control group.

Perhaps it may be intuitive that patients with headache would benefit from a multidisciplinary program which included pharmacological treatment and taught behavioral management skills. Studies have shown that relaxation techniques and cognitive behavioural therapy are beneficial to patients with headache. ${ }^{7,8}$ Therefore, one might expect that a SelfManagement program which provided education in these areas would benefit patients. While the degree of improvement due to Self-Management over and above that provided by medications cannot be determined in the present study, it has been previously reported that such behavioural interventions, especially when combined with pharmacological treatment, do produce superior benefit compared to unidisciplinary treatment approaches. For example, in a randomized, placebo-controlled study by Holroyd et $\mathrm{al}^{20}$ tricyclic prophylactic therapy, stress management training, the combination of the two, and a placebo treatment were compared in patients with chronic tension type headache. Compared to placebo, both tricyclic medication and stress management were effective therapies, but the combination of tricyclic and stress management was the most likely to produce clinically significant improvement (reduction in headache of $\geq$ $50 \%)$.

In CHAMP a large emphasis is placed on Self-Management. In Self-Management programs the patient plays a central role in the management of their illness. ${ }^{21}$ Self-Management programs provide the patient with the necessary knowledge and skills to enable them to take a more active role in managing their chronic illness. This was accomplished in CHAMP through a combination of the Education Session, which provides patients with information; the Lifestyle Assessment, which provides more in depth and tailored information; and the SelfManagement workshop, which teaches the necessary behavioural skills to effectively manage their headaches. While individually, educational programs and Self-Management programs have been shown to be beneficial to patients, ${ }^{22-24}$ the concept behind CHAMP was to combine all these facets of headache care in order to create a comprehensive program which would increase the likelihood of our patients with difficult headache problems being able to successfully manage their headaches. We have previously reported greater effectiveness for a pain-center based multidisciplinary headache program as compared to an outpatient physician-based specialty headache clinic. ${ }^{25}$ It is encouraging that the CHAMP multidisciplinary program, which is less intensive, was also able to demonstrate efficacy in a relatively refractory headache patient population. Moreover, the gains made by the patients in the present study have the potential to be long-term in that they were still present, and in fact increasing, three months after completion of the SelfManagement workshops. However, further research is needed to determine the sustainability of the gains made by patients in programs such as ours over time. Similar results have been reported for neurologist-led multidisciplinary programs for headache. One study reported maintenance of improvement at six months after therapy in a multidisciplinary program..$^{26}$

While the results of the current study are encouraging, there are limitations that should be noted. Most importantly, a control group was not available for comparison to the treatment group, and therefore we cannot isolate the effects of the behavioral treatments of the program from the medical treatments. While the patients were participating in the Education Session, the Lifestyle Assessment, and the Self Management workshop, the patients also had contact with their family physicians and in many cases the CHAMP neurologists. The results reported in the present study can only be interpreted as an evaluation of the program as a whole and not specified to one particular component of CHAMP. In order to determine the degree of 
improvement seen relating to the work of the NPHPs specifically, a randomized controlled study would need to be executed. Such studies are difficult, however, in a referral setting, as patients are referred because they are having problems coping with their headaches, and those open to Self-Management are generally eager to begin.

Based upon the results presented here and previously reported data, it is suggested that serious consideration be given to making multidisciplinary programs, with a focus on SelfManagement, more accessible to patients with difficult headache problems. There are many potential benefits, including greater reduction in headache related disability for patients, less use of scarce physician resources through greater use of non-physician health professionals, and potentially greater ability of patients to cope with their headache attacks with less use of expensive emergency room and inpatient facilities. After SelfManagement, many of our patients did report an increased ability to successfully deal with their remaining headache attacks. Such programs do have limitations, however. One of the largest obstacles is the inability of many patients to take part in SelfManagement workshops because of time constraints and distance to a site with such treatment programs. A reluctance to participate in Self-Management because of skepticism regarding its usefulness is another barrier to such programs; many patients are still searching for a medical "cure" for their headache problem, and are not willing to invest the time and effort required by non-pharmacological treatment approaches, such as SelfManagement. As a result of all of these factors, only a minority of the patients in the CHAMP program participated in the SelfManagement workshop.

It is important to comment on our patient population and the generalizability of the studied population. Our patient population was a severely affected headache population, with a mean of 18 headache days per month. More research is needed to determine whether patients with less severe headache would benefit from a multidisciplinary program such as CHAMP. It might be that such patients with less sensitization of central pain pathways would benefit even more than the population examined here. It could be further speculated that appropriate lifestyle modifications and the mastery of stress management and other Self-Management techniques in a less severe population could slow or prevent the progression of their headaches to a chronic daily headache syndrome. Migraine is very common in the general population, and obtaining the resources needed to adequately treat these patients is indeed challenging. However, it is our view that greater utilization of non-physician health care professionals in multidisciplinary programs has the potential to improve our ability to meet this challenge, given the general shortage of both primary care physicians and specialists in Canada. Ideally the present and future research which supports this view will convince healthcare funding agencies to financially support multidisciplinary health care teams to assist physicians to meet the healthcare challenge posed by the large population of patients with significant headache problems.

Many patients with difficult headache problems are currently not well served by our health care system. Our experience with CHAMP provides evidence that a multidisciplinary headache program can be successfully established as part of the Canadian public healthcare system. Our study describes a multidisciplinary headache management program which includes a selfmanagement workshop as one of its cornerstones. The multidisciplinary treatment model used in the present study combines various aspects of evidence-based behavioural interventions, maximizes cost efficiency through the use of group workshops, but does not compromise evidence-based pharmacological interventions. We suggest that educational and Self-Management components similar to that described here be incorporated into headache management programs in conjunction with pharmacological treatments in order to maximize treatment efficacy. A broad approach with multiple facets of care is likely to provide benefit to a larger proportion of patients. Further research is needed to determine the efficacy of the various components of multidisciplinary headache treatment programs.

\section{ACKNOWLEDGEMENTS}

The authors acknowledge the help of the CHAMP team: Dr. A. Eloff; Dr. L. Cooke; Irene O’Callaghan, RN; Cheryl Lake, OT; Allison McLean, OT; Kathryn Coutts, OT; and Janice Cathcart, MA(Psych), for their help in data collection and for their editorial help.

K.M.Sauro is supported through a Canadian Headache Society fellowship, sponsored by GlaxoSmithKline. W.J.Becker acts as a consultant for numerous pharmaceutical companies including GlaxoSmithKline, Pfizer, and Merck.

\section{REFERENCES}

1. Lawrence D. From Chaos to Care. Cambridge: Perseus Publishing; 2002.

2. Buchner M, Zahlten-Hinguranage A, Schiltenwolf M, Neubauer E. Therapy outcome after multidisciplinary treatment for chronic neck and chronic low back pain: a prospective clinical study in 365 patients. Scand J Rheumatol. 2006; 35(5):363-7.

3. Flor H, Fydrich T, Turk DC. Efficacy of multidisciplinary pain treatment centers: a meta-analytic review. Pain. 1992; 49(2): 221-30.

4. Guzman J, Esmail R, Karjalainen K, Malmivaara A, Irvin E, Bombardier C. Multidisciplinary rehabilitation for chronic low back pain: systematic review. BMJ. 2001; 322(7301):1511-16.

5. Scharff L, Marcus DA. Interdisciplinary outpatient group treatment of intractable headache. Headache. 1994; 34(2):73-8.

6. Lemstra M, Stewart B, Olszynski WP. Effectiveness of multidisciplinary intervention in the treatment of migraine: a randomized clinical trial. Headache. 2002; 42(9):845-54.

7. Penzien DB, Rains JC, Lipchik GL, Creer TL. Behavioral interventions for tension-type headache: overview of current therapies and recommendation for a self-management model for chronic headache. Curr Pain Headache Rep. 2004; 8(6):489-99.

8. Campbell JK. Diagnosis and treatment of cluster headache. J Pain Symptom Manage. 1993; 8(3):155-64.

9. Melzack R, Wall PD. Pain mechanisms: a new theory. Science. 1965; 150(699):971-9.

10. Jacobson GP, Ramadan NM, Aggarwal SK, Newman CW. The Henry Ford Hospital headache disability inventory (HDI). Neurology. 1994; 44(5):837-42.

11. Kosinski M, Bayliss MS, Bjorner JB, Ware JE, Jr., Garber WH, Batenhorst A, et al. A six-item short-form survey for measuring headache impact: the HIT-6. Qual Life Res. 2003; 12(8):963-74.

12. Radloff LS. The CES-D Scale: a self-report depression scale for research in the general population. Appl Psychol Measurement. $1977 ; 1: 385$.

13. The International Classification of Headache Disorders: 2nd ed. Cephalalgia. 2004; 24 Suppl 1:9-160. 
14. Silberstein SD, Lipton RB, Solomon S, Mathew NT. Classification of daily and near-daily headaches: proposed revisions to the IHS criteria. Headache. 1994; 34:1-7.

15. Fernandez E, Sheffield J. Relative contributions of life events versus daily hassles to the frequency and intensity of headaches. Headache. 1996; 36(10):595-602.

16. Andrasik F. Behavioral treatment of migraine: current status and future directions. Expert Rev Neurother. 2004; 4(3):403-13.

17. Bakal DA, Demjen S, Kaganov JA. Cognitive behavioral treatment of chronic headache. Headache. 1981; 21(3):81-6.

18. Holroyd KA, Nash JM, Pingel JD, Cordingley GE, Jerome A. A comparison of pharmacological (amitriptyline HCL) and nonpharmacological (cognitive-behavioral) therapies for chronic tension headaches. J Consult Clin Psychol. 1991; 59(3):387-93.

19. Nash JM, Park ER, Walker BB, Gordon N, Nicholson RA. Cognitive-behavioral group treatment for disabling headache. Pain Med. 2004; 5(2):178-86.

20. Holroyd KA, O'Donnell FJ, Stensland M, Lipchik GL, Cordingley GE, Carlson BW. Management of chronic tension-type headache with tricyclic antidepressant medication, stress management therapy, and their combination: a randomized controlled trial. JAMA. 2001; 285(17):2208-15.
21. Lorig KR, Sobel DS, Ritter PL, Laurent D, Hobbs M. Effect of a self-management program on patients with chronic disease. Eff Clin Pract. 2001; 4(6):256-62.

22. Harpole LH, Samsa GP, Jurgelski AE, Shipley JL, Bernstein A, Matchar DB. Headache management program improves outcome for chronic headache. Headache. 2003; 43(7):715-24.

23. Walker BB, Nash JM. Effectiveness of a multidisciplinary outpatient headache management program. Med Health RI. 2002; 85(9):269-72.

24. Rothrock JF, Parada VA, Sims C, Key K, Walters NS, Zweifler RM. The impact of intensive patient education on clinical outcome in a clinic-based migraine population. Headache. 2006; 46(5):726-31

25. Magnusson JE, Riess CM, Becker WJ. Effectiveness of a multidisciplinary treatment program for chronic daily headache. Can J Neurol Sci. 2004; 31(1):72-9.

26. Blumenfeld A, Tischio M. Center of excellence for headache care: group model at Kaiser Permanente. Headache. 2003; 43(5): 431-40. 